(C) 2019 IEEE. Personal use of this material is permitted. Permission from IEEE must be obtained for all other uses, in any current or future media, including reprinting/republishing this material for advertising or promotional purposes, creating new collective works, for resale or redistribution to servers or lists, or reuse of any copyrighted component of this work in other works. 


\section{Glossopticon: Visualising Archival Data}

\author{
Andrew Burrell \\ Faculty of Design Architecture and \\ Building \\ University of Technology Sydney \\ andrew.burrell@uts.edu.au
}

\author{
Rachel Hendery \\ Western Sydney University \\ Sydney, Australia \\ r.hendery@westernsydney.edu.au
}

\author{
Nick Thieberger \\ School of Languages and \\ Linguistics / ARC Centre of \\ Excellence for the Dynamics of \\ Language \\ University of Melbourne \\ ORCID: 0000-0001-8797-1018
}

\begin{abstract}
Glossopticon is a Virtual Reality (VR)-based information visualisation system that presents heritage audio recordings in the archival linguistic data of the Pacific and Regional Archive for Digital Sources in Endangered Cultures (PARADISEC) collection. This paper will outline the project, the way it has used VR to visualise information and how it has been used on multiple levels - from public exhibition piece, to research tool-and in doing so provides an exemplar of the notion of the collaborative, interdisciplinary project as a basic unit of scholarly investigation within the digital humanities.
\end{abstract}

Keywords - virtual reality, research through design, linguistic data, archives, information visualisation

Glossopticon (https:/glossopticon.com/) is a Virtual Reality (VR)-based information visualisation system that presents heritage audio recordings in the archival linguistic data of the Pacific and Regional Archive for Digital Sources in Endangered Cultures (PARADISEC) collection. In developing Glossopticon as a platform we were able to take advantage of some of the unique affordances made available through the medium of VR for information visualisation and come to an understanding of how designing virtual environments with these affordances in mind allowed us to target a number of distinct audiences. This paper will outline the project, the way it has used VR to visualise information and how it has been used on multiple levels - from public exhibition piece, to research tool-and in doing so provides an exemplar of the notion of the collaborative, interdisciplinary project as a basic unit of scholarly investigation within the digital humanities [1].

While the experience differs across versions of the project, the following description will be useful in the context of this ongoing discussion. When using the system, a user moves across a representation of a geographic region, such as Vanuatu, or Papua New Guinea and is surrounded by spatialised audio of the nearby languages. The location of each language is represented by a pillar of light surrounded by a semi-transparent dome the size of which corresponds to the approximate number of speakers. A HUD (heads up display) overlay ${ }^{1}$ can be switched on and off, which shows the amount and diversity of archival materials (grammar, lexicon, multimedia, etc) and a documentation score for the language at which the user is gazing. In one experimental version of this system, when the user gazes at a language for long enough, the language is 'bookmarked' and a link back to its entry in the archives is added to a personal webpage generated for the user to visit after they exit the VR experience, in an attempt to bridge the ephemeral virtual space and the persistent real world (cf. [2] for discussion of other approaches to this problem).

The project was initially conceived of as a virtual reality exhibition for a museum display (Glossopticon v1) using the HTC Vive [3]. It has evolved into a larger project using both WebVR and the Unity game engine as development environment, and is now open to other data sources. This has allowed other researchers to visualise their own information, using it as a tool for the research and presentation of information beyond what was originally envisaged.

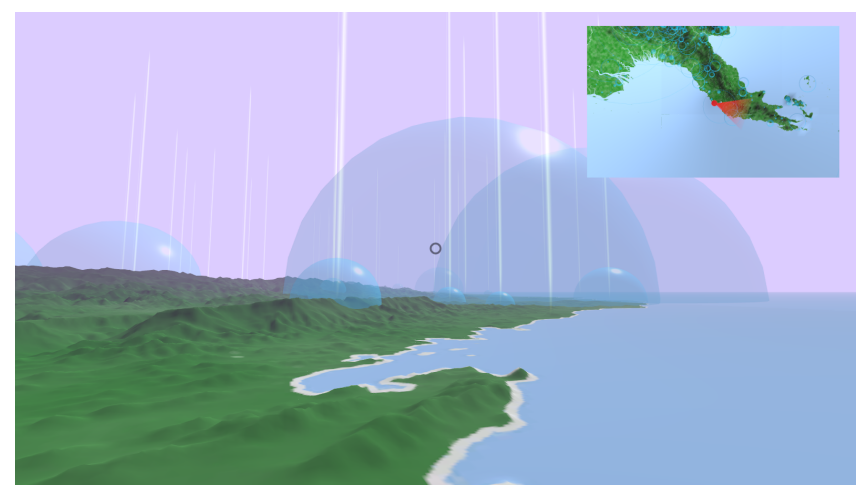

Figure 1: Glossopticon 2017

\footnotetext{
${ }^{1}$ A HUD, or heads up display, is a 2D overlay of information displayed in front of the viewer's near depth of view, attached their relative position in space, as opposed to being located at a fixed position in the virtual space itself.
} 
Our central concern, however, was always visualising the PARADISEC collection, an archive which holds records of 1,202 languages in various forms, most of which include audio recordings. There are nearly 9,700 hours of recordings, too large a set to listen to and make sense of. Of course, a standard metadata set is applied to these items, but for many heritage recordings there is little description available. Glossopticon is a means of exploring this vast amount of material by taking metadata from the catalogue and playing a snippet of the audio associated with it. This metadata was also supplemented with data drawn from the OLAC (Open Language Archives Community) visualiser [4], to show how much information is held in all OLAC archives for any given language. The use of virtual reality as an interface to a library or archive is something that has long been anticipated [5], but generally conceptualised as a reproduction of library or archive shelves [6], or of documents and images from the archive in the virtual space. Rather than recreating a virtual archive, we wanted to bring the archival materials back to life and connect users more directly with the environment in which the materials were recorded. The most similar precedent to this is perhaps the State Library of Western Australia's PAV 3D project, which displays the library's image archives in a virtual geographical environment, using a 360-degree cylinder [7].

In this paper we discuss the various versions of this project that have been developed since this initial version in 2017, which include the creation of an entirely new version in WebVR, designed for use with mobile phones and 'cardboard' style headsets, the development of a backend that is easily extensible to new regions of the world and new datasets, as well as the open-sourcing of the project. Burrell and Hendery are currently also developing another related piece of software that includes the original data but allows the layering of it with various kinds of other datasets onto a more abstract map. In Table 1 we show how the different pieces of software discussed here relate to each other. Across this spectrum of audiences, the source of information remains the same, though experientially for the end user, the information can be read as data or experienced as capta [8] depending on their own needs as a viewer or user of the system.

The WebVR version (item 3, in Table 1) is offered as a community project [9] that others can populate with their own data, with the possibility of a map on which language samples can be heard from around the globe. A possible use of this system is also as a front-end to the catalog of another archival collection.

This WebVR version of Glossopticon has been open sourced and made publically available at https://github.com/clavis-magna/glossopticon-webvr. It allows users to drop the following into a spreadsheet: language name, language id, geography (point), audio snippet link, and metadata including documentation score, number of speakers and country. A web link to addition information can also be included that a user may "collect" for later viewing by visiting a separate web page that formats these links a list. The audio file itself can be similarly uploaded. The system allows for the inclusion of bounding box limits within the data set, which can be represented in the visualisation - although we found this visualisation to be overly taxing of mobile phone hardware. While we experimented with using Google Firebase (https://firebase.google.com/) as a backend for the project to enable this service, we ultimately found that although it provided a robust and stable solution, it created an unnecessary barrier for entry, whereas reading directly from a formatted data file exported from a spreadsheet and placed in a web directory, allowing any user with a basic web knowledge the means of easily using their own data.

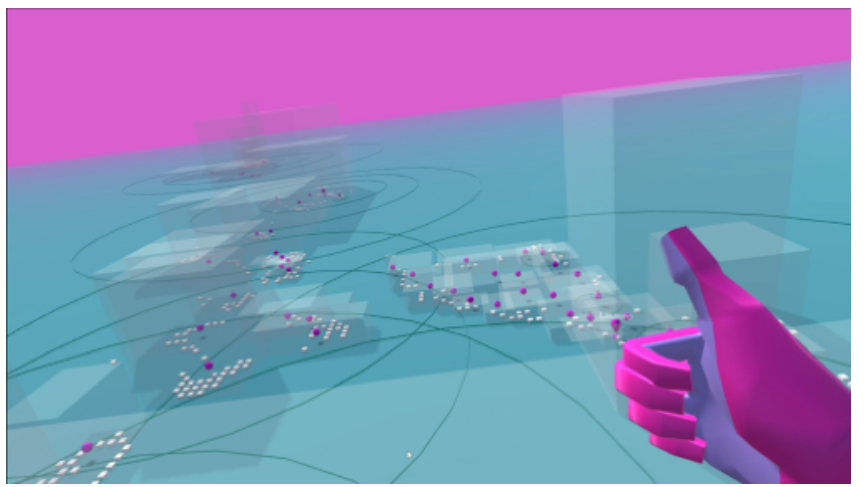

Figure 2: An early version of Layered Horizons

The metadata presented in all versions of Glossopticon includes a documentation index drawn from OLAC's listing of information per language. A separate project [4] takes OLAC data and infers a documentation score based on how many items there are in each of OLAC's categories [Language descriptions; Lexical resources; Primary texts; Other resources about the language]. This then gives an idea of what little archived information there is for most languages. We display icons of different colours representing a scale of three (fewer than 20 resources; between 20 and 150 resources; more than 150 resources).

Glossopticon continues to have as a central aim to create an environment that is broadly accessibility and can be successfully used on a wide variety of platforms, from phone and browser-based VR to high end VR equipment. One of the advantages of moving back to the Unity game engine as development environment, is the "build once, deploy everywhere" framework that allows export of the project to multiple platforms from the same project with only minor alterations necessary. Unity also provides an accessible development environment that allows project collaborators with little to no coding knowledge to work directly on the realisation of the project.

We have also chosen Unity as the development environment for 'Layered Horizons' (item 4 in Table 1), we are also implementing hand gesture-based interaction using 
the Leap Motion ${ }^{2}$, to enhance the affordances of VR in creating an environment where embodied cognition is central to interactions and understanding of data. Initial user feedback on this system has indicated that it is a much more intuitive way of interacting with the environment, than one of navigating with off-the-shelf game controllers. The splitting of the software into the various different development paths described above means that we are separating out a public outreach and archive discovery tool, which retains the more realistic landscape and a research tool which is more of a 'datascape' than a landscape and allows the archive data to be layered with other kinds of data from other sources relating to the languages and/or the region.

Lev Manovich notes that information visualisation is the representation of datasets in such a way as to reveal structure [10], what we have been developing with the 'Layered Horizons' iteration of the project is a movement towards a visualisation approach to that reveals linguistic structures by bringing together both linguistic and non-linguistic data into the same visualisation. As pointed out by Sara Diamond, "A successful visualization may cross multiple boundaries and provide different perspectives on the same data set [11]" we aim to reveal new perspectives across multiple data sets.

Significantly, one key aspect that each of the versions has in common is the way in which spatialised audio is used to allow a user access to the audio snippets gleaned from PARADISEC. From the very first showing of the project and through to current iterations, audiences have made clear that this is one of their most memorable takeaways of the experience. They enjoy being physically immersed in an ocean of voices that can be navigated (and indeed used to navigate) and which can be isolated via proximity to their origin in space. This proximity is also the key to providing access to the embedded metadata.

Additionally, because of the distance between points on the map we included a location teleport function, allowing users to jump to a predefined series of locations rather than navigating to them through continuous movement, which also allowed us to curate a series of locations where we felt a lay-user would discover some of the most visually and sonically interesting locations with the visualisation.

One of the new developments in 'Layered Horizons' (item 4, Table 1) is to use point-based Pacific places that allow navigation via hand gestures using Leap. This geographic data is derived from the "files of geographic names information" databases of The National GeospatialIntelligence $\mathrm{Agency}^{3}$, which provide coordinates, names and

\footnotetext{
2 The Leap Motion is a small piece of hardware that allows very accurate skeletal hand tracking and it is the authors' view that real time, natural, gesture based interaction using hand (and in the future, body) tracking will become one of the standard means of interaction in VR.

https://www.leapmotion.com/

${ }^{3}$ Toponymic information is based on the Geographic Names Database, containing official standard names approved by the United States Board on Geographic Names and maintained by the National Geospatial-Intelligence
}

other details of named geographic locations. By using the coordinates to build a point-based map of the Pacific regions in question, we will be able to manipulate the landscape programmatically, for example to grow or shrink islands according to their importance in particular datasets. The user can make gestures for interacting with datapoints - cradling a language point in a hand presents the user with related metadata, while pointing at a location reveals the language name and potentially other important data. Navigation is also via gesture: a finger pointed forward allows the user to "fly" in the direction they are facing, while a thumbs up or down, allows them to float up or down in space. We have been conducting user testing around these interactions as part of a wider suite of user feedback data collection ${ }^{4}$ and initial results are indicating that these natural hand-based interactions allow a user a more immediately understandable relationship with the virtual environment and their place in it as an active participant/observer.

As the various projects described above are developed, we aim to make them available as tools that we hope are useful to others. In order to avoid confusion between our own ongoing research and the tools as outcomes, moving forward the "Glossopticon" title will be associated with the original tool and new projects built off it will take on their own identity with new titles, as has been the case for "Layered Horizons". We will continue to work towards sharing and open sourcing core components of the ongoing projects. As has been stated we have provided the source for the WebVR version on Github [9] as a shareable outcome many of the core aspects are represented in this code, so it will be helpful to anyone wanting to experiment with it within the AFrame WebVR framework, or bring it back to Unity themselves. As new/related projects in Unity are developed to a stage where they are ready for sharing and opening up, they will also be made available - for example, "Layered Horizons" with the Leap Motion integration will be shared publicly in the very near future.

\section{BIBLIOGRAPHY}

[1] A. Burdick, J. Drucker, P. Lunenfeld, T. Presner and J. Schnapp, "The Project as Basic Unit," in Digital Humanities, Cambridge, MIT Press, 2012, pp. 124-126.

[2] K. Martin, B. Greenspan and A. Quan-Haase, "STAK Serendipitous tool for augmenting knowledge: A conceptual tool for bridging digital and physical resources," Digital Studies / Le champ numérique, vol. 9, 2017.

[3] N. Thieberger and R. Hendery, "Augmented Reality for Visualizing Archive Records," in ICLDC5, Manoa, Hawai'i., 2017.

[4] N. Thieberger, "Language Archives data visualisation,"

Agency. More information is available at the Maps and Geodata link at www.nga.mil. The National Geospatial-Intelligence Agency name, initials, and seal are protected by 10 United States Code Section 425.

${ }^{4}$ Andrew Burrell and Rachel Hendery, Glossopticon VR user testing and feedback, UTS HREC REF NO. ETH18-2293, in progress. 
OLAC, 2018. [Online]. Available: http://www.languagearchives.services/about/olac-vis. [Accessed 1203 2019].

[5] A. Pouter, "Towards a virtual reality library," Aslib Proceedings, vol. 1, no. 11-17, p. 45, 1993.

[6] A. Chow, C. Baity, P. Chappell, D. Rachlin, C. Vinson and M. Zamarripa, "When real and virtual worlds collide: a public library's management of a second life library," in ALA Virtual Communities and Libraries Membership Interest Group online conference, 2010.

[7] A. Lugmayr, A. Greenfeld, A. Woods and P. Joseph, "Cultural Visualisation of a Cultural Photographic Collection in 3D Environments," in Entertainment Computing - ICEC 2016, Vienna, Austria, 2016.

[8] J. Drucker, Graphesis: Visual Knowledge Production and Representation, Cambridge: Massachusetts Harvard University Press, 2014.
[9] A. Burrell and R. Hendery, "Glossopticon VR," 2018. [Online]. Available: https://github.com/clavismagna/glossopticon-webvr. [Accessed 1103 2019].

[10] L. Manovich, What is visualization?, Poetess Archive Journal $2.1,2010$.

[11] S. Diamond, Lenticular Galaxies: The Polyvalent Aesthetics of Data Visualization, C Theory. Code Drift: Essays in Critical Digital Studies, 2010.

\begin{tabular}{|c|c|c|c|c|}
\hline Name & Platform & Primary Audience & Description & Date \\
\hline (1) Glossopticon 2017 & Unity (for HTC Vive) & General public & $\begin{array}{l}\text { Realistic topographic terrain-based } \\
\text { landscape }\end{array}$ & 2017 \\
\hline (2) Glossopticon iPad & Unity (for iPad) & General public & $\begin{array}{l}\text { Realistic topographic terrain-based } \\
\text { landscape }\end{array}$ & 2017 \\
\hline $\begin{array}{l}\text { (3) Glossopticon } \\
\text { WebVR }\end{array}$ & $\begin{array}{l}\text { WebVR (for phones with } \\
\text { Cardboard-style headsets, } \\
\text { Desktop, or HTC Vive) }\end{array}$ & $\begin{array}{l}\text { General public / } \\
\text { developers }\end{array}$ & $\begin{array}{l}\text { Customisable 2D image as indicator of } \\
\text { landscape, open, extensible backend }\end{array}$ & 2018 \\
\hline (4) Layered Horizons & $\begin{array}{l}\text { Unity (for HTC Vive with } \\
\text { Leap Motion) }\end{array}$ & Researchers & $\begin{array}{l}\text { More abstract, generative, 'datascapes', } \\
\text { gesture-controlled }\end{array}$ & ongoing \\
\hline
\end{tabular}

TABLE I. GLOSSOPTICON VERSIONS 